HELSINGIN YLIOPISTO

HELSINGFORS UNIVERSITET 


\section{PD-L1 expression in endometrial carcinoma cells and intratumoral immune cells:}

\section{2 differences across histological and TCGA-based molecular subgroups}

3 Annukka Pasanen $\mathrm{MD}^{1,2}$, Terhi Ahvenainen $\mathrm{MSc}^{3,4}$, Teijo Pellinen $\mathrm{PhD}^{5}$, Pia Vahteristo $\mathrm{PhD}^{3,4}$, Mikko

4 Loukovaara $\mathrm{MD}, \mathrm{PhD}^{6}$, Ralf Bützow $\mathrm{MD}, \mathrm{PhD}^{1,2,6}$

5

$6 \quad{ }^{1}$ Department of Pathology, University of Helsinki and Helsinki University Hospital, Helsinki, Finland.

$7 \quad{ }^{2}$ Applied Tumor Genomics Research Program, University of Helsinki, Helsinki, Finland

$8{ }^{3}$ Department of Medical and Clinical Genetics, Medicum, University of Helsinki, Helsinki, Finland.

$9{ }^{4}$ Genome-Scale Biology Research Program, Research Programs Unit, University of Helsinki, Helsinki,

10 Finland.

$11 \quad{ }^{5}$ Institute for Molecular Medicine Finland (FIMM), Helsinki, Finland.

$12{ }^{6}$ Department of Obstetrics and Gynecology, University of Helsinki and Helsinki University Hospital,

13 Helsinki, Finland.

15 Corresponding author:

16 Annukka Pasanen, Department of Pathology, University of Helsinki and Helsinki University Hospital,

17 Haartmaninkatu 3, 00290 Helsinki, Finland. Electronic address: annukka.pasanen@hus.fi

24 Disclosures: The author(s) have no conflicts of interest or funding to disclose. 


\section{Abstract}

29 PD-L1 is a biomarker that may predict the response to antiPD-1/PD-L1 immunotherapy. We 30 evaluated the expression of PD-L1 in carcinoma cells and immune cells across

31 histopathological and TCGA molecular subgroups of endometrial carcinoma.

32 Our study included 842 patients with endometrial carcinoma. Direct sequencing of polymerase 33 epsilon (POLE) exonuclease domain hot spots and conventional immunohistochemistry 34 (MLH1, PMS2, MSH2, MSH6, p53) were conducted to identify TCGA classification-based 35 molecular subgroups of endometrial carcinoma: POLE-mutated, mismatch repair (MMR) 36 deficient, no specific molecular profile and p53-aberrant. Multiplex immunohistochemistry was

37 performed to evaluate PD-L1 expression in carcinoma cells $(\mathrm{Ca})$ and tumor-infiltrating immune 38 cells (ICs). PD-L1 expression in carcinoma cells and in ICs was detected in $8.6 \%$ and $27.7 \%$ 39 of the cases, respectively. Combined positive score (CPS) was $i ̈ 1 \%$ in $19.4 \%$ of the samples. 40 PD-L1 positivity in carcinoma cells, ICs and CPS correlated with tumor T cell density (TILs, $41 \mathrm{p}<0.001)$. POLE-mutated and MMR-deficient tumors were more likely to present PD-L1 42 expressing ICs, CPS positivity and abundant TILs compared with other TCGA subgroups 43 ( $p<0.001)$. No differences existed in Ca-PD-L1 expression $(p=0.366)$. Within various 44 histotypes, non-endometrioid carcinomas displayed the highest Ca-PD-L1, ICs and CPS 45 ( $\mathrm{p}<0.03)$. Advanced cancers showed more frequent Ca-PD-L1 positivity $(\mathrm{p}=0.016)$, CPS $46(\mathrm{p}=0.029)$ and ICï $1 \%(\mathrm{p}=0.037)$ positivity compared to early disease.

47 In conclusion, PD-L1 expression profiles differ between molecular subclasses, histological 48 subtypes and disease stage of endometrial carcinoma. Prospective studies are needed to explore 49 the predictive value of various PD-L1 scoring systems within the subgroups of endometrial 50 cancer. CPS presents methodological advantages over cell-type specific scoring systems. 
52 Key words: endometrial carcinoma, PD-L1, TCGA classification

\section{INTRODUCTION}

55 Immune checkpoint inhibitors have emerged as a promising treatment option for various types

56 of cancer, but their potential in endometrial cancer (EC) is unknown (1). Immunotherapy

57 enhances the immune system's innate potential to fight cancer cells. An effective anti-tumor

58 response relies primarily on the capability of $\mathrm{T}$ cells to recognize tumor-derived peptides as

59 non-self. Typically, these immunogenic peptides are produced as a consequence of mutations

60 and, accordingly, tumors with a high mutational burden are presumed to be highly

61 immunogenic. Immune responses are controlled by various pathways that tumor cells may

62 exploit to escape immune surveillance. One of the main immunosuppressive pathways is the

63 PD-1/PD-L1 interaction taking place between $\mathrm{T}$ cell programmed death 1 (PD-1) receptor and

64 its ligand PD-L1 located on various types of cells, including immune cells and carcinoma cells

65 (2). Antibody-mediated blockade of the PD-1/PD-L1 immune checkpoint has been shown to

66 reverse T-cell inactivation exposing tumor cells to immune attack (3). Anti-PD-1/PD-L1

67 therapies have produced impressive treatment outcomes in patients with non-small cell lung

68 cancer, melanoma and renal cell carcinoma $(1,4)$. Limited-sample studies have obtained

69 promising results also with endometrial carcinoma patients (5,6). Also other immune

70 checkpoints (e.g. CTLA-4, LAG-3) may induce immunosuppression in tumors $(7,8)$.

71 Patients tolerate immunotherapy well compared to standard chemotherapy, but side effects may

72 occur. The therapy is costly and not all patients benefit from it. Thus, an efficient use of

73 immunotherapy requires appropriate patient selection strategies. Often the selection of patients

74 to anti-PD-1/PD-L1 therapy is guided by PD-L1 immunohistochemical (IHC) assays. Scoring

75 methods and adopted cut-offs vary according to the tumor type and individual anti-PD-1/PD- 
76 L1 agents. Proposed scoring algorithms evaluate PD-L1 positivity in carcinoma cells and/or

77 immune cells separately or in combination (combined positive score, CPS) (9-11). Reported

78 frequencies of PD-L1 positivity in endometrial carcinoma vary considerably (0.9-44.3\%) even

79 in unselected EC cohorts (Table 1) (12-24). Such variability may in part derive from different

80 antibody clones and different cut-offs. In fact, notable interassay variation has been reported

81 within commercially available PD-L1 immunohistochemical assays $(25,26)$. Accuracy of IHC

82 scorings may also suffer from problems related to traditional chromogenic PD-L1

83 immunohistochemistry. Staining of the tumor cells may be weak and unspecific cytoplasmic

84 staining occurs. Moreover, intratumoral T cells and macrophages often present membranous

85 staining and they may be misinterpreted as carcinoma cells (27). Multiplex IHC overcomes

86 these limitations by simultaneous detection of a biomarker and numerous cell-specific markers

87 on a single paraffin tissue section, allowing the identification and quantification of various cell

88 types expressing the antigen of interest (28).

89 Endometrial carcinoma is not a uniform disease entity, as it comprises various histological and

90 molecular subgroups, each with their own clinicopathological characteristics. Given this

91 heterogeneity, exhaustive biomarker studies rely on well-powered subclass analyses. The goal

92 of our study was to explore PD-L1 expression and T cell inflammation within histological

93 subtypes and TCGA-based molecular subgroups of endometrial cancer. Fluorescent multiplex

94 immunohistochemistry was performed to overcome limitations related to traditional

95 immunohistochemical evaluation.

97 MATERIALS AND METHODS

98 Patients who underwent primary surgical treatment for endometrial cancer at the Department 99 of Obstetrics and Gynecology, Helsinki University Hospital, between January 1, 2007, and 
100 December 31, 2012, were identified $(n=965)$. Patients with adequate tumor samples for a tissue

101

102

103

104

105

106

107

108

109

110

111

112

113

114

115

116

117

118

119

120

121

122

123

124 microarray (TMA) were included in the study $(n=842)$. Approvals of the Institutional Review Board and the National Authority for Medicolegal Affairs of Finland were obtained. Clinical data were collected from institutional medical records. Lacking follow-up data were obtained from Statistics Finland or completed by contacting primary physicians at the referring institutions.

We performed immunohistochemistry on multicore tissue microarray (TMA) slides, prepared as described before (29). The following monoclonal antibodies were used for chromogenic immunohistochemistry: MLH1 (ES05, Dako), PMS2 (EPR3947, Epitomics), MSH-2 (G2191129, BD Biosciences), MSH-6 (EPR3945, Abcam), p53 (DO-7, Dako) and PD-L1 (SP263, Ventana). TMA slides were scanned with 3-dimensional Histech Pannoramic 250 Flash II scanner by Fimmic Oy (Helsinki, Finland). Slide images were managed and analyzed with WebMicroscope Software (Fimmic Oy). Virtual slides were scored by a pathologist blinded to clinical data. A second investigator examined equivocal cases and a consensus was reached. Mismatch repair protein status was considered deficient (MMRd) when we observed a complete loss of nuclear expression in carcinoma cells of one or more MMR proteins (MLH1, PMS2, MSH2, MSH6) detected by immunohistochemistry. Aberrant p53 staining was defined as strong and diffuse nuclear staining or completely negative ('null') staining in carcinoma cells. Weak and heterogeneous staining was classified as wild type expression. Stromal cells and inflammatory cells served as internal control for MMR and p53 stainings. Samples with scarce carcinoma cells or with completely negative staining of the internal control (when applicable), were discarded. Representative images of MLH-1 and p53 staining patterns are shown in Figure 1.

The fluorescent multiplex immunohistochemistry was carried out as described by Blom et al. with following modifications (30). Primary antibodies were: PD-L1 (CST, E1L3N), CD3 
125 (Thermo, MA5-14482), CD163 (Abcam, ab188571), and PanEpi (cocktail of anti-PanCk, C-

126 11, Abcam, Ab77531; anti-PanCk AE1/AE3, InVitrogen, 180132; E-cadherin, BD clone 36).

127 Nuclei were stained using DAPI (Roche). Five-channel fluorescent images were acquired using

128 Metafer 5 scanning and imaging platform (MetaSystems, Alltlussheim, Germany) equipped

129 with AxioImager Z2 microscope with a 20x objective (Carl Zeiss, Goettingen, Germany) and a

130 CoolCube 2m CCD camera (MetaSystems, Alltlussheim, Germany). The image analysis was

131 carried out both visually by a pathologist and by a cell image analysis software (CellProfiler

132 version 2.2.0). Scoring was primarily performed by a pathologist and in rare equivocal cases

133 automated image analysis was used to support the decision-making. Necrotic areas and scarce

134 samples (<100 cells) were excluded from scoring. PD-L1 expression was defined as partial or

135 complete membranous staining in carcinoma cells and membranous and/or cytoplasmic

136 staining in immune cells (CD3-positive T lymphocytes and CD163-positive macrophages

137 within tumor nests and/or adjacent supporting stroma). We determined the percentage of

138 positive carcinoma cells and immune cells separately and in combination. To calculate the

139 combined positive score (CPS), we divided the total number of PD-L1-positive cells (carcinoma

140 cells, lymphocytes, and macrophages) by the number of viable carcinoma cells, multiplied by

141100 (9). Semiquantitative scoring was adopted as follows: 0: <1\% of the cells; 1: 1-4\%; 2: 5-

$1429 \%$; 3: 10-49\%; 4: ï 50\%. The cut-off for positive PD-L1 staining was set at 1\%. The cut off

143 for strong positivity was set according to the results of a previous randomized trial (i $50 \%$ for

144 carcinoma cells and $\mathbf{1} 10 \%$ for immune cells) (31). Comparative images of conventional

145 chromogenic immunohistochemistry and multiplex immunofluorescence of PD-L1 positive and

146 negative cells are shown in Figure 2. Tumoral CD3+ lymphocytic infiltration (TILs) was semi-

147 quantitatively scored as scarce, moderate or abundant.

148 For DNA extraction, representative areas of formalin-fixed paraffin-embedded tumor tissue

149 were macrodissected as identified by pathologist assessment. DNA was extracted by proteinase 
$150 \mathrm{~K} /$ phenol-chloroform method. POLE exonuclease domain mutation screening of hot spots in 151 exon 9 (c.857C>G, p.P286R; c.890C>T, p.S297F), exon 13 (c.1231G>C, p.V411L) and exon 15214 (c.1366G>C, p.A456P), was performed by direct sequencing. The following primers were 153 used: $\quad$ Ex 9F $\left(5^{\prime}-3^{\prime}\right):$ CCTAATGGGGAGTTTAGAGCTT; $\quad$ Ex $9 \mathrm{R} \quad\left(5^{\prime}-3^{\prime}\right)$ : 154 CCCATCCCAGGAGCTTACTT; Ex 13F (5'-3’): TCTGTTCTCATTCTCCTTCCAG; Ex 155 13R (5'-3'): CGGGATGTGGCTTACGTG; Ex 14F (5'-3’): TGACCCTGGGCTCTTGATTT; 156 Ex 14R (5'-3'): ACAGGACAGATAATGCTCACC. PCR products were sequenced on an 157 ABI3730xl Automatic DNA Sequencer at Institute for Molecular Medicine Finland (FIMM), 158 Helsinki. Sequence graphs were analyzed both manually and with Mutation Surveyor 159 (Softgenetics, State College, PA). Only cases with good-quality sequence for all the examined 160 POLE hot spots were included in the analysis.

161 Pearson $\$ 2$ test and Fisher exact test were used for comparisons of categorical variables. Survival 162 curves were calculated by the Kaplan-Meier method. A log-rank test was used to test for survival 163 differences. Disease-specific survival was defined as the time from date of surgery to death from 164 endometrial cancer. Statistical significance was set at $p<0.05$. Cohen's kappa statistics were 165 calculated to measure the agreement between multiplex and chromogenic immunohistochemistry 166 for PD-L1. Based on kappa references outlined by Landis and Koch, the strength of agreement 167 was considered fair for kappa values between 0.21 and 0.40 and moderate for kappa values 168 between 0.41 and 0.60 (32). Data were analyzed using IBM SPSS version 25 software (IBM 169 Corp., Armonk, New York, USA).

\section{RESULTS}

172 Clinicopathological characteristics of the study cases are summarized in Table 2. Of the 842 173 patients included in the study, $745(88.5 \%)$ had endometrioid and 97 (11.5\%) non-endometrioid 174 carcinoma. Median follow-up of patients was 78 months (range 1 to 136 months). Sequencing 
of all the targeted genomic regions of POLE was successful for 553 cases. POLE mutation was

176 detected in $7.4 \%$ of endometrioid carcinomas and $4.0 \%$ of non-endometrioid carcinomas $(6.7 \%$

177 of all cases). MMR protein deficiency was found in $37.7 \%$ of endometrioid carcinomas and

$17825.6 \%$ of non-endometrioid carcinomas (36.2\% of all the cases). Aberrant p53 profile was

179 detected in $10.8 \%$ of endometrioid tumors and $61.9 \%$ of non-endometrioid tumors (16.8\% of

180 all the samples). A minority of cases displayed multiple molecular features. Both POLE

181 mutation and aberrant p53 expression were present in $0.4 \%$ of the cases and both POLE

182 mutation and MMR protein deficiency in $0.2 \%$ of the patients. Only one sample $(0.2 \%)$ had all

183 three molecular alterations. These patients were allocated into the POLEmut molecular

184 subgroup. Both MMR deficiency and aberrant p53 status were detected in $3.1 \%$ of the cases.

185 These were classified as MMRd tumors.

186 In the multiplex immunofluorescence staining, 8.6\% of the cases presented PD-L1 expression 187 on carcinoma cells (Caï $1 \%$ ) and $27.7 \%$ on intratumoral immune cells (ICsï 1\%). CPS was 188 positive (CPSï 1\%) in 19.4\% of the samples. High PD-L1 expression (Caï 50\% or ICsï 10\%) 189 was observed in $0.5 \%$ and $8.6 \%$ of the cases, respectively. Relative frequencies of 190 semiquantitative staining scores are presented in Table 3. Tumors with moderate-abundant T 191 cell density presented PD-L1 positivity in carcinoma cells (10.6\%), ICs (36.6\%) and CPS $192(26.8 \%)$ more frequently than tumors with scarce lymphocytic infiltration $(\mathrm{Ca} 5.6 \%, \mathrm{p}=0.019$;

193 ICs $14.6 \%, \mathrm{p}<0.001$, CPS 8.4\%, $\mathrm{p}<0.001)$. Concomitant presence of moderate-abundant $\mathrm{T}$ cell 194 infiltrates and any PD-L1 positivity ("T cell inflamed PD-L1 positive" phenotype), was 195 observed in $25.1 \%$ of all the tumors.

196 Relative frequencies of PD-L1 positivity in carcinoma cells varied significantly between 197 histotypes: endometrioid carcinoma $8.0 \%$, clear cell carcinoma $14.7 \%$, serous carcinoma $3.7 \%$, 198 undifferentiated carcinoma $14.7 \%$ and carcinosarcoma 20\% ( $\mathrm{p}=0.022$, Figure 3). Observed 199 relative frequencies of CPSï $1 \%$ were: endometrioid carcinoma 17.1\%, clear cell carcinoma 
$38.2 \%$, serous carcinoma $37.0 \%$, undifferentiated carcinoma $42.9 \%$ and carcinosarcoma $26.7 \%$

201 ( $\mathrm{p}<0.001$; Figure 3). Similar differences were noted in the immune cell expression of PD-L1 with significantly higher relative frequencies of expression in the non-endometrioid carcinomas $(\mathrm{p}=0.028)$. By contrast, we found no statistically significant differences between histological subgroups and strong PD-L1 positivity in ICs ( $\mathrm{p}=0.148$, Figure 3 ). Our cohort included only one neuroendocrine carcinoma, which presented PD-L1 expression on both carcinoma cells and immune cells. We found no correlation between Ca-PD-L1 expression and grade of differentiation of endometrioid carcinomas (G1-2 vs G3, p=0.08), whereas CPS and ICï 10\% PD-L1 expression were more frequent in G3 as compared to G1-2 endometrioid carcinomas (33.0\% vs $14.3 \%$ and $20.8 \%$ vs $5.4 \%$, respectively, $\mathrm{p}<0.001)$. The overall quantity of CD3+

210 TILs (scarce-moderate vs abundant) did not differ significantly in histological subgroups $211 \quad(\mathrm{p}=0.158)$ or between grade of differentiation of endometrioid carcinoma $(\mathrm{p}=0.722)$.

PD-L1 expression profiles were also analyzed according to FIGO 2009 stage of disease (stage I-II vs III-IV, Figure 3). Samples from patients with advanced stage (III-IV) disease were more 214 likely to present Ca-PD-L1 positivity ( $13.6 \%$ vs $7.5 \%, \mathrm{p}=0.016)$, CPS (25.9\% vs $18.0 \%$, $\mathrm{p}=0.029)$ and ICï $1 \%$ (34.7\% vs $26.2 \%, \mathrm{p}=0.037)$ positivity as compared to early stage (I-II) disease. Differences in the ICï 10\% ( $\mathrm{p}=0.270)$ or the overall quantity of TILs $(\mathrm{p}=0.598)$ were

217 not statistically significant. In advanced disease, strong Ca-PD-L1 positivity was found in $1.4 \%$ 218 of the cases and strong IC positivity in $10.9 \%$ of the cases.

219 Samples with successful POLE sequencing and immunohistochemical stainings of MMR proteins and p53 (512 cases), were stratified into TCGA-based molecular subclasses. POLEmut and MMRd tumors exhibited higher relative frequencies of immune cell PD-L1 positivity (55.9\% and 40.9\%) and CPS positivity (44.1\% and 29.6\%) compared to NSMP (IC: $13.9 \%$,

223 CPS: 9.1\%) and p53ab cases $(25.4 \%, 20.9 \%$; p <0.001, Figure 4). Significant differences were observed also for strong positivity in ICs ( $\mathrm{p}<0.001$, Figure 4). POLEmut and MMRd cases were 
also more likely to present abundant intratumoral $\mathrm{T}$ cell infiltrates $(26.5 \%$ and $27.8 \%$ respectively) compared to NSMP and p53ab cases $(15.3 \%$ and $16.7 \%$ respectively; $\mathrm{p}=0.014)$.

227 Similarly, we observed "T cell inflamed PD-L1 positive" phenotype more frequently in

228

229

230

231

232

233

234

235

236

237

238

239

240

241

242

243

244

245

246

247 POLEmut and MMRd groups (50.0\% and 34.9\%, respectively) compared to other TCGA subclasses $(16.3 \%$ and $17.9 \%$; $\mathrm{p}<0.001)$. PD-L1 expression in carcinoma cells showed no correlation with TCGA classification $(\mathrm{p}=0.366)$.

In Kaplan Meier analysis, disease specific survival segregated by histotype and TCGA subgroups as expected (pî 0.001, data not shown). POLEmut group had excellent outcomes (no disease related deaths in this group) and aberrant p53 status associated with poor disease specific survival. Scarce overall quantity of TILs predicted poor prognosis ( $p=0.001$ ), whereas PD-L1 expression on carcinoma cells, ICs or CPS showed no correlation with outcome $(\mathrm{p}=0.298, \mathrm{p}=0.592, \mathrm{p}=0.569$, respectively $)$.

According to kappa statistics, multiplex and chromogenic immunohistochemistry scorings showed moderate agreement for CPS (kappa 0.540) and poor agreement for PD-L1 expression in carcinoma cells (kappa 0.279).

\section{DISCUSSION}

In the evolving era of personalized medicine, immunotherapy offers new treatment options for cancer patients. FDA has approved mismatch repair deficiency/microsatellite instability as selection criteria for anti PD-1/PD-L1 therapy (33). Treatment indications in mismatch repair stable EC and the role of biomarkers, including PD-L1, have remained unsettled. To facilitate prospective studies, we profiled PD-L1 expression across histopathological and TCGA molecular subgroups of endometrial carcinoma. 
248 In our study cohort, $8.6 \%$ of the cases presented PD-L1 expression in carcinoma cells and

$24927.7 \%$ in the ICs. In line with previous studies, PD-L1 expression on carcinoma cells or on

250 lymphocytes showed no correlation with survival $(18,19)$. Non-endometrioid carcinomas were

251 more likely to present PD-L1 positive carcinoma cells, CPS and ICs compared to endometrioid

252 carcinomas. In the subgroup of endometrioid ECs, high grade of differentiation was associated

253 with more frequent CPS and IC positivity compared to low grade disease.

254 In a landmark study, The Cancer Genome Atlas (TCGA) identified 4 distinct molecular 255 subgroups of endometrial carcinoma: POLE ultramutated, microsatellite instability 256 hypermutated (MSI-H), copy-number-low microsatellite stable (MSS), and copy-number-high 257 (34). Vast majority (90\%) of the copy-number-high tumors presented TP53 mutations.

258 Consequently, TP53 mutational analysis or immunohistochemical analysis of p53 expression 259 have been proposed as a surrogate marker for this subgroup of tumors $(35,36)$. POLE mutated 260 tumors are characterized by defects in the proof-reading function of DNA polymerase epsilon 261 and harbor the highest rate of somatic mutations, followed by MSI-H tumors characterized by 262 defects in DNA mismatch repair activity. These highly mutated tumors have been reported to 263 contain a large number of predicted neoantigens and activated cytotoxic tumor infiltrating $\mathrm{T}$ 264 lymphocytes, often expressing PD-1 and PD-L1 (13,17,24,37,38). Corroborating these 265 findings, we observed significantly higher relative frequencies of heavy $\mathrm{T}$ cell infiltrates and 266 PD-L1 expressing ICs in the POLE mutated and MMR deficient groups compared to other 267 TCGA subgroups. By contrast, we found no correlation between Ca-PD-L1 expression and the 268 molecular subclasses.

269 It has been speculated, that tumors most likely to respond to PD-1/PD-L1 blockade 270 characteristically present an "adaptive resistance" phenotype (T cell inflamed PD-L1 positive 271 phenotype, i.e. concomitant presence of intratumoral T cell infiltrates and PD-L1 positivity) 272 (39-41). Consequently, based on previous studies and our results, POLEmut and MMRd tumors 
273 become natural candidates for immune checkpoint blockade therapy. Interestingly, in a phase

274 II study of an anti-PD-1 agent in patients with various types of advanced cancer (including 275 endometrial carcinoma), mismatch-repair status itself, and not PD-L1 expression, predicted 276 clinical benefit (42).

277 Clinical studies suggest a correlation between increasing levels of PD-L1 expression and drug 278 efficacy, but definite scoring systems and cut-offs may be tumor-specific and still need to be 279 determined $(43,44)$. Most trials focus on PD-L1 expression on carcinoma cells. Nonetheless, 280 various studies report associations between clinicopathological characteristics of EC and PD281 L1 expression on immune cells rather than tumor cells (13-15,21). The significance of these 282 correlations is unknown. In a trial including multiple cancer types, PD-L1 positivity on tumor283 infiltrating immune cells, but not on tumor cells, predicted response to cancer treatment with an anti-PD-L1 agent, MPDL3280A (atezolizumab) (45). Accordingly, for atezolizumab treatment, expression in intratumoral immune cells (IC) is also used as an indicator for potential response (46). In a randomized lung cancer trial, patients with tumors expressing high levels of

287 PD-L1 (defined as Caï 50\% or ICï 10\%) derived the greatest benefit from atezolizumab treatment (31). We observed high tumoral Ca-PD-L1 expression in only $0.5 \%$ of the tumors. However, strong IC positivity (ï 10\%) was seen in $8.6 \%$ of the cases. The need for alternative treatment options is greatest in advanced stage (III-IV) endometrial carcinoma, which presented

291 with stronger Ca-PD-L1, IC and CPS expression levels than early cancers.

292 Intratumoral heterogeneity of protein expression may lead to decreased sensitivity in TMA 293 studies. Clonal loss of MMR protein expression has been reported and it is not known whether 294 focal MMR deficiency could invoke a PD-L1 response in a predominantly intact tumor. 295 However, the rate of mismatch repair deficiency in our study was not lower than generally 296 reported in the literature. In a study by Sloan et al., heterogeneous PD-L1 positivity in ECs 297 typically consisted of individual cells or small clusters of cells, that were fairly evenly 
distributed throughout the tumor (18). Further, previous studies have shown that TMAs with

299 three core biopsies per tumor adequately represent the tumor phenotype, even with antigens

300 known to be heterogeneous $(47,48)$. Since performing MMR or PD-L1 stainings on whole

301 sections was not feasible for this vast cohort, to improve sensitivity, we included 4 tissue cores

302 from each tumor in our TMA. We have previously demonstrated a high concordance between

303 our TMA and the corresponding whole sections, as concerns expression of L1CAM, a highly

304 heterogeneous antigen (29). As an advantage, TMA methodology allowed us to analyze a large

305 number of cases by multiplex IHC and conventional standardized immunohistochemistry

306 (Ventana clone SP263).

307 In concordance analysis of multiplex IHC and conventional IHC, carcinoma cell proportion

308 score showed only fair agreement, which in part reflects the difficulty of differentiating PD-L1

309 positive carcinoma cells from macrophages in the chromogenic IHC. Accordingly, moderate agreement was found between CPS scorings. In some cases, chromogenic immunostainings

311 presented equivocal staining in the stromal compartment, which may have led to false positivity

312 in IC scoring and CPS. Multiplex immunohistochemistry aptly circumvented these limitations.

313 Some of the differences between the staining results may be explained by the low cut off for

314 PD-L1 positivity (1\%) and the use of different PD-L1 antibody clones, i.e. E1L3N for multiplex and SP263 for chromogenic IHC. In our experience, multiplex immunohistochemistry clearly outperforms traditional IHC when analyzing PD-L1 expression in various cell types. However,

317 at the moment it cannot be adopted in routine diagnostics and the problems related to cell-type 318 specific scoring systems may be circumvented using a scoring method that combines positivity 319 of both carcinoma and intratumoral immune cells. Based on our results, the correlation of such score (CPS) to clinicopathological characteristics of endometrial carcinoma is equal or better

321 than score based on carcinoma cells only. 
322 In conclusion, we identified differences in PD-L1 expression between histological subtypes,

323 disease stage and TCGA-based molecular subgroups of endometrial carcinoma. PD-L1

324 positivity was more frequently observed in intratumoral immune cells compared to carcinoma

325 cells. Based on our results, prospective trials should consider not only PD-L1 expression on

326 carcinoma cells but also immune cells, when stratifying patients with endometrial carcinoma

327 for immunotherapy. Combined scoring systems may present methodological advantages over

328 cell-type specific scoring. Further studies are necessary to explore the predictive value of this

329 differential expression of PD-L1, various scoring methods and the applicability of

330 immunotherapy in different subgroups of endometrial cancer.

331

332

333

334

References

335

336 1. Brahmer JR, Tykodi SS, Chow LQ, et al. Safety and activity of anti-PD-L1 antibody in 337 patients with advanced cancer N Engl J Med 2012;366:2455-2465.

338 2. Freeman GJ, Long AJ, Iwai Y, et al. Engagement of the PD-1 immunoinhibitory receptor

339 by a novel B7 family member leads to negative regulation of lymphocyte activation $J$ Exp

340 Med 2000;192:1027-1034.

341 3. Fife BT, Pauken KE, Eagar TN, et al. Interactions between PD-1 and PD-L1 promote

342 tolerance by blocking the TCR-induced stop signal Nat Immunol 2009;10:1185-1192.

343 4. Garon EB, Rizvi NA, Hui R, et al. Pembrolizumab for the treatment of non-small-cell lung 344 cancer N Engl J Med 2015;372:2018-2028. 
345 5. Ott PA, Bang YJ, Berton-Rigaud D, et al. Safety and Antitumor Activity of Pembrolizumab

346 in Advanced Programmed Death Ligand 1-Positive Endometrial Cancer: Results From the

347 KEYNOTE-028 Study J Clin Oncol 2017;35:2535-2541.

348 6. Le DT, Durham JN, Smith KN, et al. Mismatch repair deficiency predicts response of solid 349 tumors to PD-1 blockade. Science 2017;357:409-413.

350 7. Pardoll DM. The blockade of immune checkpoints in cancer immunotherapy Nat Rev

$351 \quad$ Cancer 2012;12:252-264.

352 8. Nirschl CJ, Drake CG. Molecular pathways: coexpression of immune checkpoint

353 molecules: signaling pathways and implications for cancer immunotherapy Clin Cancer Res

$354 \quad 2013 ; 19: 4917-4924$.

355 9. www.agilent.com/cs/library/usermanuals/public/29219_pd-11-ihc-22C3-pharmdx-gastric-

356 interpretation-manual_us.pdf

357 10. www.ventana.com/documents/PD-L1_SP142-NSCLC-Brochure.pdf

358 11. www.ventana.com/documents/PD-L1_SP142-UC-Brochure.pdf

359 12. Herzog TJ, Arguello D, Reddy SK, et al. PD-1, PD-L1 expression in 1599 gynecological

360 cancers: Implications for immunotherapy. Gynecologic Oncology 2015;137:204-205.

361 13. Howitt BE, Shukla SA, Sholl LM, et al. Association of Polymerase e-Mutated and

362 Microsatellite-Instable Endometrial Cancers With Neoantigen Load, Number of Tumor-

363 Infiltrating Lymphocytes, and Expression of PD-1 and PD-L1. JAMA Oncol2015;1:1319-

3641323.

365 14. Eggink FA, Van Gool IC, Leary A, et al. Immunological profiling of molecularly classified

366 high-risk endometrial cancers identifies POLE-mutant and microsatellite unstable carcinomas

367 as candidates for checkpoint inhibition. Oncoimmunology 2016;6:e1264565.

368 15. Mo Z, Liu J, Zhang Q, et al. Expression of PD-1, PD-L1 and PD-L2 is associated with differentiation status and histological type of endometrial cancer Oncol Lett 2016;12:944-950. 
370 16. Bregar A, Deshpande A, Grange C, et al. Characterization of immune regulatory

371 molecules B7-H4 and PD-L1 in low and high grade endometrial tumors Gynecol Oncol $372 \quad 2017 ; 145: 446-452$.

373 17. Yamashita H, Nakayama K, Ishikawa M, et al. Microsatellite instability is a biomarker for 374 immune checkpoint inhibitors in endometrial cancer Oncotarget 2017;9:5652-5664.

375 18. Sloan EA, Ring KL, Willis BC, et al. PD-L1 Expression in Mismatch Repair-deficient

376 Endometrial Carcinomas, Including Lynch Syndrome-associated and MLH1 Promoter

377 Hypermethylated Tumors Am J Surg Pathol 2017;41:326-333.

378 19. Li Z, Joehlin-Price AS, Rhoades J, et al. Programmed Death Ligand 1 Expression Among

379700 Consecutive Endometrial Cancers: Strong Association With Mismatch Repair Protein

380 Deficiency. Int J Gynecol Cancer 2018;28:59-68.

381 20. Kim J, Kim S, Lee HS, et al. Prognostic implication of programmed cell death 1 protein 382 and its ligand expressions in endometrial cancer Gynecol Oncol 2018;149:381-387.

383 21. Asaka S, Yen TT, Wang TL, et al. T cell-inflamed phenotype and increased Foxp3

384 expression in infiltrating T-cells of mismatch-repair deficient endometrial cancers Mod Pathol $385 \quad 2019 ; 32: 576-584$.

386 22. Crumley S, Kurnit K, Hudgens C, et al. Identification of a subset of microsatellite-stable 387 endometrial carcinoma with high PD-L1 and CD8+ lymphocytes Mod Pathol 2019;32:396388404.

389 23. Kucukgoz Gulec U, Kilic Bagir E, Paydas S, et al. Programmed death-1 (PD-1) and 390 programmed death-ligand 1 (PD-L1) expressions in type 2 endometrial cancer Arch Gynecol 391 Obstet 2019;.

392 24. Talhouk A, Derocher H, Schmidt P, et al. Molecular Subtype Not Immune Response 393 Drives Outcomes in Endometrial Carcinoma Clin Cancer Res 2018; 
394 25. McLaughlin J, Han G, Schalper KA, et al. Quantitative Assessment of the Heterogeneity 395 of PD-L1 Expression in Non-Small-Cell Lung Cancer. JAMA Oncol 2016;2:46-54.

396 26. Hirsch FR, McElhinny A, Stanforth D, et al. PD-L1 Immunohistochemistry Assays for 397 Lung Cancer: Results from Phase 1 of the Blueprint PD-L1 IHC Assay Comparison Project $J$ 398 Thorac Oncol 2017;12:208-222.

399 27. Brunnstrom H, Johansson A, Westbom-Fremer S, et al. PD-L1 immunohistochemistry in 400 clinical diagnostics of lung cancer: inter-pathologist variability is higher than assay variability. 401 Mod Pathol 2017;30:1411-1421.

402 28. Parra ER, Uraoka N, Jiang M, et al. Validation of multiplex immunofluorescence panels 403 using multispectral microscopy for immune-profiling of formalin-fixed and paraffin-embedded 404 human tumor tissues. Sci Rep 2017;7:13380-017-13942-8.

405 29. Pasanen A, Tuomi T, Isola J, et al. L1 Cell Adhesion Molecule as a Predictor of Disease406 Specific Survival and Patterns of Relapse in Endometrial Cancer Int J Gynecol Cancer $407 \quad 2016 ; 26: 1465-1471$.

408 30. Blom S, Paavolainen L, Bychkov D, et al. Systems pathology by multiplexed

409 immunohistochemistry and whole-slide digital image analysis Sci Rep 2017;7:15580-017$410 \quad 15798-4$.

411 31. Rittmeyer A, Barlesi F, Waterkamp D, et al. Atezolizumab versus docetaxel in patients 412 with previously treated non-small-cell lung cancer (OAK): a phase 3, open-label, multicentre 413 randomised controlled trial. The Lancet 2017;389:255-265.

414 32. Landis JR, Koch GG. The measurement of observer agreement for categorical data. 415 Biometrics 1977;33:159-174.

416 33. www.fda.gov/newsevents/newsroom/pressannouncements/ucm560167.htm

417 34. Cancer Genome Atlas Research Network, Kandoth C, Schultz N, et al. Integrated

418 genomic characterization of endometrial carcinoma Nature 2013;497:67-73. 
419 35. Stelloo E, Bosse T, Nout RA, et al. Refining prognosis and identifying targetable

420 pathways for high-risk endometrial cancer; a TransPORTEC initiative. Modern Pathology

$4212015 ; 2015 ; 28: 836$ <last_page $>844$.

422 36. Talhouk A, McConechy MK, Leung S, et al. A clinically applicable molecular-based

423 classification for endometrial cancers. Br J Cancer 2015;113:299-310.

424 37. Dolcetti R, Viel A, Doglioni C, et al. High Prevalence of Activated Intraepithelial Cytotoxic

425 T Lymphocytes and Increased Neoplastic Cell Apoptosis in Colorectal Carcinomas with

426 Microsatellite Instability. The American Journal of Pathology 1999;154:1805-1813.

427 38. Pakish JB, Zhang Q, Chen Z, et al. Immune Microenvironment in Microsatellite-Instable

428 Endometrial Cancers: Hereditary or Sporadic Origin Matters. Clin Cancer Res 2017;23:4473-

4294481.

430 39. Tumeh PC, Harview CL, Yearley JH, et al. PD-1 blockade induces responses by

431 inhibiting adaptive immune resistance Nature 2014;515:568-571.

432 40. Teng MW, Ngiow SF, Ribas A, et al. Classifying Cancers Based on T-cell Infiltration and 433 PD-L1 Cancer Res 2015;75:2139-2145.

434 41. Althammer S, Tan TH, Spitzmuller A, et al. Automated image analysis of NSCLC

435 biopsies to predict response to anti-PD-L1 therapy. J Immunother Cancer 2019;7:121-019-

436 0589-x.

437 42. Le DT, Uram JN, Wang H, et al. PD-1 Blockade in Tumors with Mismatch-Repair

438 Deficiency N Engl J Med 2015;372:2509-2520.

439 43. Borghaei H, Paz-Ares L, Horn L, et al. Nivolumab versus Docetaxel in Advanced

440 Nonsquamous Non-Small-Cell Lung Cancer N Engl J Med 2015;373:1627-1639.

441 44. Herbst RS, Baas P, Kim DW, et al. Pembrolizumab versus docetaxel for previously

442 treated, PD-L1-positive, advanced non-small-cell lung cancer (KEYNOTE-010): a

443 randomised controlled trial. Lancet 2016;387:1540-1550. 
45. Herbst RS, Soria JC, Kowanetz M, et al. Predictive correlates of response to the anti-PD-

L1 antibody MPDL3280A in cancer patients. Nature 2014;515:563-567.

46. www.gene.com/download/pdf/tecentriq_prescribing.pdf

47. Fons G, Hasibuan SM, van der Velden J, et al. Validation of tissue microarray technology

in endometrioid cancer of the endometrium. J Clin Pathol 2007;60:500-503.

\section{LEGENDS}

Table 1. PD-L1 expression in endometrial carcinoma: overall frequency of PD-L1 positivity (tumoral and immune cells, CPS), cut-offs for positive staining and significant correlations between PD-L1

Figure 1. MLH-1 and p53 immunohistochemistry: a) Endometrial carcinoma cells exhibiting positive nuclear MLH-1 staining, b) Loss of MLH-1 expression in carcinoma cells with

$\mathrm{END}=$ endometrioid, $\mathrm{NE}=$ non-endometrioid, $\mathrm{MSI}=$ microsatellite instable, MSS=microsatellite stable, MMRd/p=mismatch repair deficient/proficient, Chr=chromogenic immunostaining, CA=carcinoma cells, IC=immune cells, $\mathrm{CPS}=$ combined positive score, mut=mutated, $\mathrm{LVI}=$ lymphovascular invasion, $\mathrm{LN}+=$ lymph nodal metastasis, $\mathrm{MI}=$ myometrial invasion; $\mathrm{NS}=$ non-significant, $\mathrm{PFS}=$ progression-free survival, $\mathrm{OS}=$ overall survival; *values extracted from graphs p53, e) aberrant p53 (diffuse overexpression), f) aberrant p53 (null), stromal cells serving as internal control.

Figure 2. PD-L1 positive (a,b) and negative (c,d) endometrial carcinoma: a,c) PD-L1, 474 (carcinoma cells, white), CD3 (T cells, green), CD163 (macrophages, red). Note the co475 localization of PD-L1 and epithelial or immune cell markers: PD-L1 positive carcinoma cells 476 (light blue), lymphocytes (turquoise) and macrophages (magenta)

478 Figure 3. Frequency of PD-L1 positivity in carcinoma cells (ï 1\%), ICs (ï 10\%) and CPS 479 (i $1 \%$ ) according to histological subgroups ( $\mathrm{p}=0.022, \mathrm{p}=0.148$ and $\mathrm{p}<0.001$, respectively) and 480 FIGO 2009 stage ( $\mathrm{p}=0.037, \mathrm{p}=0.270$ and $\mathrm{p}=0.029$, respectively). $\mathrm{Ca}=$ carcinoma cells, $481 \mathrm{ICs}=$ immune cells, CPS=combined positive score 
483 Figure 4. Frequency of PD-L1 positivity in carcinoma cells ( $p=0.366)$, ICs ( $<<0.001)$, CPS

$484(\mathrm{p}<0.001)$ and presence of heavy $\mathrm{T}$ cell infiltrates $(\mathrm{p}=0.014)$ according to molecular

485 subgroups. POLEmut $=$ mutated $P O L E$, MMRd $=$ MMM deficient, $\mathrm{NSMP}=$ no specific

486 molecular type, p53ab = p53 aberrant. $\mathrm{Ca}=$ carcinoma cells, ICs=immune cells,

487 CPS=combined positive score

488 


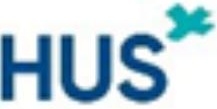

